\title{
Diagnostic Dilemma in a Case of Genital Tuberculosis
}

\author{
Ooha Thadiboina1, Deepti Shrivastava ${ }^{2}$, Harsha Shekawath ${ }^{3}$, Sandhya Pajai ${ }^{4}$, Sourya Acharya ${ }^{5}$
}

\begin{abstract}
${ }^{1}$ Department of Obstetrics and Gynaecology, Datta Meghe Institute of Medical Sciences (Deemed to be University) Sawangi, Meghe, Wardha, Maharashtra, India. ${ }^{2}$ Department of Obstetrics and Gynaecology, Datta Meghe Institute of Medical Sciences (Deemed to be University) Sawangi, Meghe, Wardha, Maharashtra, India. ${ }^{3}$ Department of Obstetrics and Gynaecology, Datta Meghe Institute of Medical Sciences (Deemed to be University) Sawangi, Meghe, Wardha, Maharashtra, India. ${ }^{4}$ Department of Obstetrics and Gynaecology, Datta Meghe Institute of Medical Sciences (Deemed to be University) Sawangi, Meghe, Wardha, Maharashtra, India. ${ }^{5}$ Department of General Medicine, Datta Meghe Institute of Medical Sciences (Deemed to be University) Sawangi, Meghe, Wardha, Maharashtra, India.
\end{abstract}

\section{INTRODUCTION}

Extrapulmonary tuberculosis accounts for a significant proportion of tuberculosis cases worldwide. Nevertheless, the diagnosis is often delayed or under dilemma or even missed due to insidious clinical presentation and unpredictable sensitivity of diagnostic tests. Here we describe a case of a 30-year-old woman of secondary amenorrhoea who was evaluated and posted for diagnostic hysterolaparoscopy and endometrial biopsy. The tubercular lesions identified on laparoscopy and endometrial tissue sampled was sent for TB-PCR, AFB culture and histopathology. TB-PCR and AFB culture reports were negative for tuberculosis, while her histopathology report was positive. She was started on category 1 anti-tubercular therapy. Her further course was uneventful and she started having normal menstrual cycles 9 months later.

It is difficult to clinically diagnose a patient of genital tuberculosis, as it ids asymptomatic in most cases. Patients with genital tuberculosis are usually young women diagnosed during workup for infertility.[1] Genital tuberculosis is usually an indolent infection with symptoms such as infertility (44\%), chronic pelvic pain $(25 \%)$, bleeding per vaginum $(18 \%)$, amenorrhoea $(5 \%)$, discharge per vaginum $(4 \%)$ and postmenopausal bleeding $(2 \%)^{[2,3]}$ In almost all of the cases, the salpinx forms the primary focus, followed by uterus and ovaries in $50-60 \%$ and $20-30 \%$ respectively. ${ }^{[4]}$

The diagnostic tests commonly performed include complete blood count, erythrocyte sedimentation rate, Mantoux test, chest radiograph, pelvic and abdominal ultrasonography, hysterosalpingography, hysteroscopy, laparoscopy, mycobacterial culture, histopathology, TB-PCR, nucleic acid amplification tests.

PCR is a molecular biological test for detecting mycobacteria in pulmonary as well as extra pulmonary samples from suspected tuberculosis patients. It is also sensitive and specific and yields results quickly. It is capable of detecting fewer than ten bacilli per $\mathrm{ml}$ of the specimen and takes only 1-2 days for the report.[5,6] Even though histopathological mode of examination is inexpensive, non-cumbersome and provides results relatively quick, seldom, if ever, are any TB bacilli seen. Secondary nature of genital tuberculosis could be the reason behind this. Traditionally, the definitive diagnosis of tuberculosis depends on identifying the causative organism, Mycobacterium tuberculosis, by acid-fast staining or by demonstrating its growth on Lowenstein Jensen medium. Microscopic examination of acid-fast bacilli (AFB) requires the presence of at least 10000 organisms per milli litre of the sample, while culture is more sensitive, requiring as little as 100 organisms per milli litre.[7] However, mycobacterium tuberculosis may take up to 8 weeks to grow in Lowenstein-Jensen medium. ${ }^{[8]}$ Substantial number of tubercular lesions in the genital tract are bacteriologically mute. On account of this and the inadvertent technical drawbacks, laboratory demonstration of growth of mycobacterium tuberculosis is difficult.
Corresponding Author: Ooha Thadiboina,

Department of Obstetrics and Gynaecology, Datta Meghe Institute of Medical Sciences (Deemed to be University) Sawangi, Meghe, Wardha, Maharashtra, India.

E-mail:drooha92@gmail.com

DOI: $10.14260 /$ jemds/2020/265

Financial or Other Competing Interests: None.

How to Cite This Article:

Thadiboina O, Shrivastava D, Shekawath $H$, et al. Diagnostic dilemma in a case of genital tuberculosis. J. Evolution Med. Dent. Sci. 2020;9(14):1223-1226, DOI: $10.14260 /$ jemds/2020/265

Submission 06-12-2019,

Peer Review 14-03-2020,

Acceptance 21-03-2020,

Published 06-04-2020.

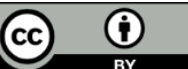


Hence, the diagnosis of extra pulmonary tuberculosis can be baffling, owing to paucibacillary load in the biological specimens, compelling a high index of suspicion. Continuing search is needed to find simpler and practicable methods for making definitive diagnosis in respect to female genital tuberculosis. Here, we focus on the limitations of various diagnostic techniques and diagnostic dilemma associated with TB-PCR, histopathology and AFB culture.

\section{PRESENTATION OF CASE}

A 30-year-old nulligravida presented at OPD of Acharya Vinoba Bhave Rural Hospital with history of amenorrhoea since 8 years. She had been married for 6 years, and was eager to conceive since then. She had her first menses at 13 years of age and her cycles were at regular intervals with average flow and duration. There was no family history of tuberculosis.

On examination, she was well built, vitals were normal, on per abdominal examination her abdomen was soft with no guarding, tenderness or rigidity. Her Mantoux test after $72 \mathrm{hrs}$. revealed wheal diameter of $6 \mathrm{~mm}$, chest x ray was normal, ultrasonography showed evidence of bilateral tubo ovarian mass. She was admitted for further evaluation with a plan of diagnostic hysterolaparoscopy and endometrial biopsy. They were both strongly suggestive of genital tuberculosis.

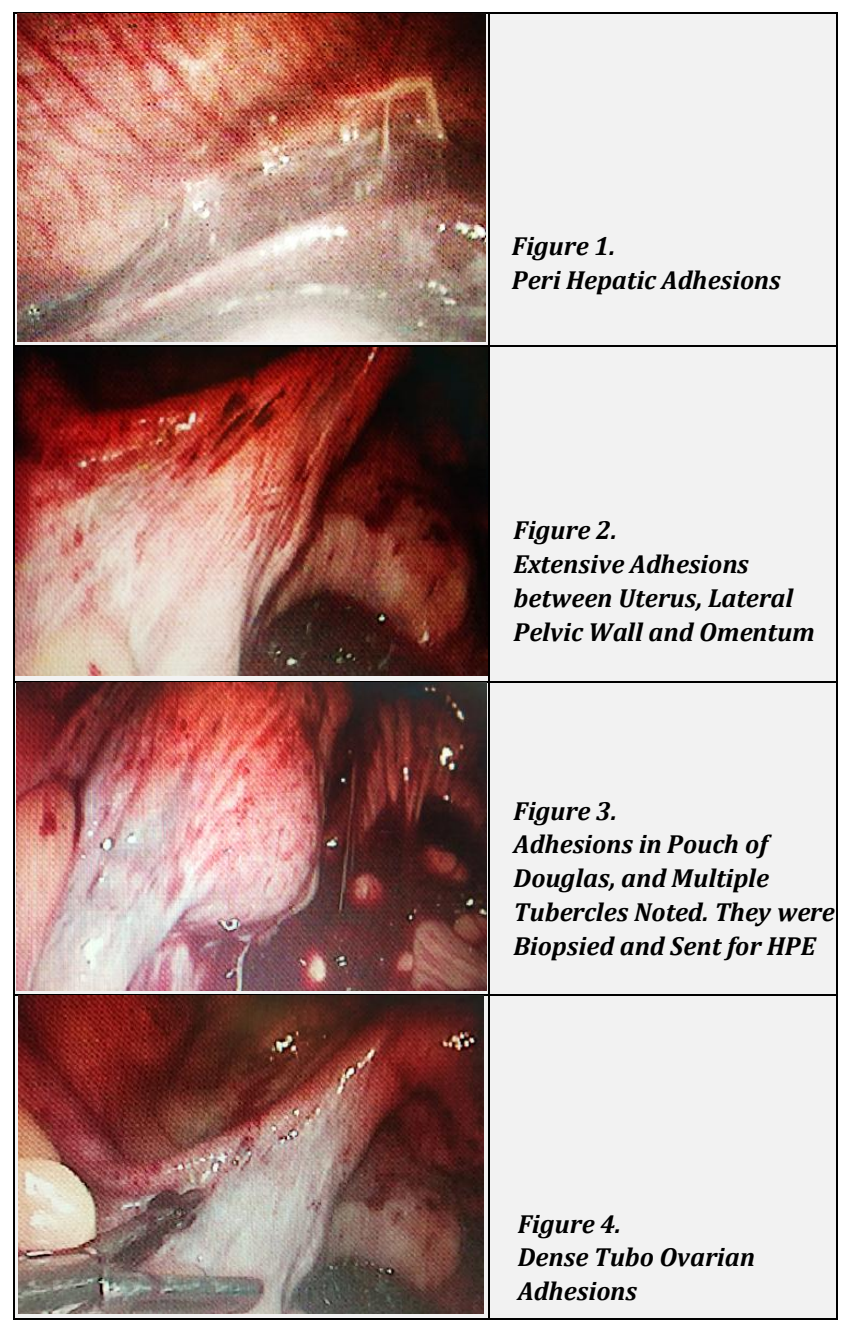

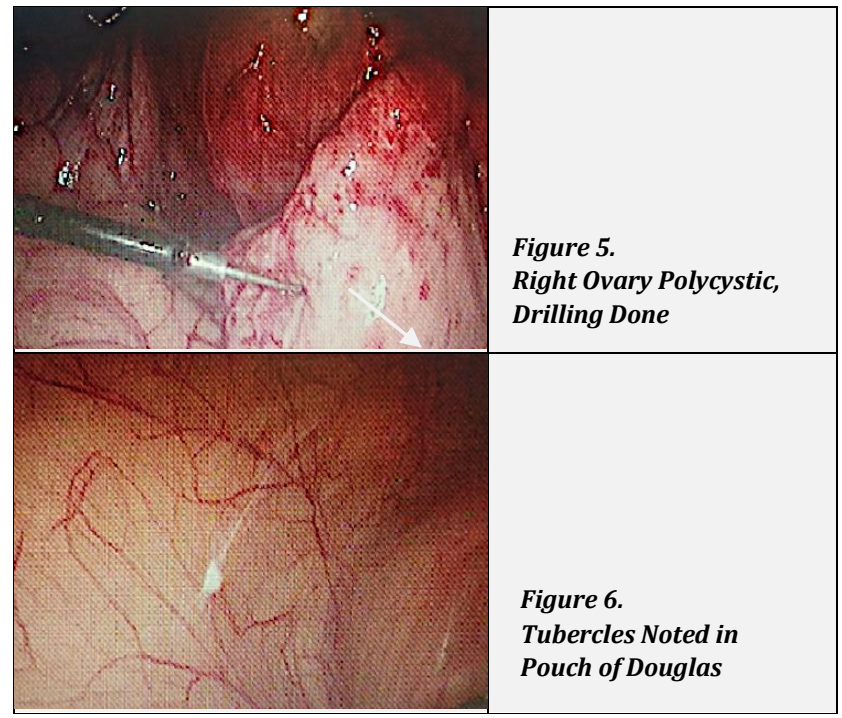

\section{Laparoscopic Findings}

- Uterus-was of normal size.

- Bilateral fallopian tube and ovaries had dense adhesions.

- Adhesions were present in pouch of Douglas; bowel was adhered to pouch of Douglas.

- Left side tubo ovarian adhesions were released.

- Right ovary was polycystic, ovarian drilling was done.

- Perihepatic adhesions were present.

- Miliary spots were found in pouch of Douglas.

\section{Hysteroscopic Findings}

- Complete septum was present in uterine cavity.

- Left ostia were visualized.

- Right ostia could not be visualized due to fibrosis.

- Intra cavitary adhesions were present.

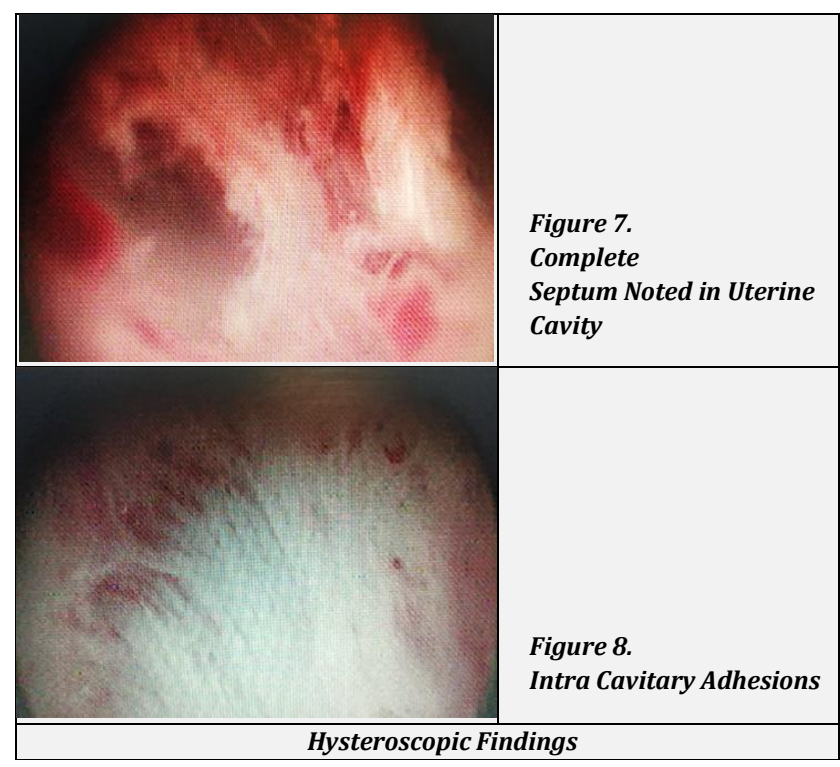

Endometrial biopsy taken was sent for TB-PCR, AFB culture and histopathology. Histopathology report showed presence of caseating granulomas surrounded by epithelioid 
cells, lymphocytes, plasma cells and giant cells and was positive for tuberculosis. TB-PCR and AFB culture were negative for tuberculosis.

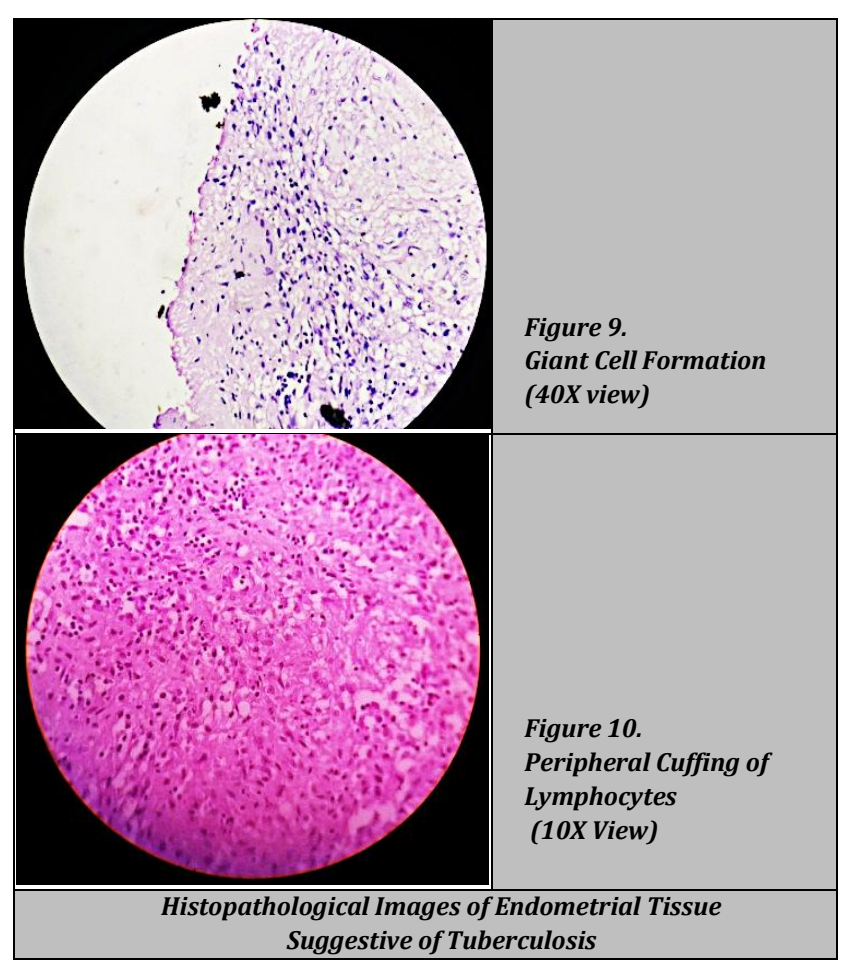

\section{DISCUSSION OF MANAGEMENT}

Patient was started on category 1 anti-tubercular therapy, which is 2 Months of HRZE (isoniazid, rifampicin, pyrazinamide and ethambutol) in the intensive phase, followed by 4 months of HR (isoniazid and rifampicin) in the continuation phase, on follow up she had regular menses 9 months after starting anti-tubercular therapy.

\section{PATHOLOGICAL DISCUSSION}

Tuberculosis of the female genital tract is not a rare entity in developing countries, but it is a challenge to make a definitive diagnosis. Genital tuberculosis can occur at any age, seventy five percent of the incidence is noted in the 20-45 years age bracket. Infertility in genital tuberculosis is due to endometrial and salpingeal pathology interfering with implantation and transportation of sperm and zygote. Other important manifestations of genital tuberculosis are menstrual irregularities like oligomenorrhoea and hypomenorrhoea. Other significant pathological findings in genital tuberculosis are Asherman's syndrome which leads to menstrual irregularities, infertility.

A high index of suspicion along with extensive investigations is of paramount importance to make a positive diagnosis. Routine laboratory investigations yield little value. A high erythrocyte sedimentation rate and positive Mantoux test are non-specific. Chest radiological studies are normal in most cases.[5] Absolute diagnosis cannot be made with certainty based on the features noted in hysterosalpingogram, laparoscopy. They still are important for retrieving tissue sample for culture and histopathological examination. $[9,10,11]$ Ultrasonography, computed axial tomography and magnetic resonance imaging are performed in scenarios where mass is felt clinically.[12]

Laparoscopic picture pointing towards tuberculosis include tubercles, caseous granulomas and beaded appearance of tubes. Laparoscopic picture of tubercles, thickened tubes, caseation in the fallopian tubes, and hydrosalpinx especially in the fimbrial end, tubo ovarian masses, flimsy adhesions in the rectouterine pouch indicate chronic tuberculosis. Histopathology reveals typical picture of caseous lesions, granulomas with giant epitheloid cells. Polymerase chain reaction (PCR) allows direct identification of mycobacterium in clinical specimens. Identification of mycobacterium tuberculosis through culture is presently the yardstick for diagnosis of genital tuberculosis. Presence of a bacteriostatic substances which inhibit the growth of bacilli could be the reason why culture positivity is rarely noted in extrapulmonary tuberculosis. ${ }^{[13]}$

PCR is a very sensitive diagnostic tool which yields rapid results. It cannot by itself confirm or exclude the diagnosis of genital tuberculosis. PCR assays target a $65 \mathrm{kDa}$ proteinencoding gene, the IS6110 element and the mpt 64 gene and other gene elements. Bhanu et al have reported PCR sensitivity of 76.9 per cent in aspiration samples and biopsies taken from endometrium. ${ }^{[14]}$ PCR false positivity rate of 9.5 percent was reported in studies conducted by R. B. P. Thangappah, C. N. Paramasivan and Sujatha Narayanan at tuberculosis research centre in Chennai.[15] PCR positivity with negative clinical findings, histopathology and culture reports may be seen in latent infections, early stages of infection with low bacilli counts, where bacilli are detected by PCR even though patients are still asymptomatic and structural damage has not yet occured. Thangappah et al have reported 7.14 percent sensitivity and 100 percent specificity of AFB culture in a study conducted on 49 cases of genital tuberculosis in Chennai.

PCR positive and BACTEC (culture) and smear negative cases could be explained by the fact that even though smear and culture have remained a gold standard in diagnosis of pulmonary TB, the very low sensitivity of culture for diagnosis of paucibacillary disease limits its practical utility in extrapulmonary TB.

In advanced cases where the endometrium was significantly destroyed and adequate endometrial tissue could not be retrieved, PCR was often negative. In such cases, TBPCR was non-representative either because of inadequate samples or because it was old, healed disease. In a study conducted by Thangappah, C. N. Paramasivan and Sujatha Narayanan at tuberculosis research centre in Chennai PCR was tested negative in 12 amongst the 28 patients whose clinical and laparoscopic imaging resembled genital tuberculosis. This points towards the possible false negative results with PCR. PCR report was negative for 2 cases who were tested positive by culture and histopathology.[16] The study conducted by Rozati et al yielded similar results.[16] Paucibacillary nature of genital tuberculosis, exact site of sample retrieved not containing any tubercular bacteria could possibly explain the high false negative rates of PCR. The specimen taken may also contain inhibitors of PCR, there by contributing to false negativity. Restrepo et al have stated that high human genome: 
bacterial genome ratio, at least 190:1, compromises mycobacterial DNA amplification.[17] Endometrial tissue samples are often mixed with blood, this contributes to false negativity of PCR. [18]

Histopathological mode of examination is inexpensive, non-cumbersome and provides results relatively quick. Owing to the secondary nature of the disease seldom, if ever, are any TB bacilli seen, as the organism load is low, the sample that is picked up need not necessarily include the infected. Moreover, as the endometrium cyclically sheds, granuloma formation is not flavoured, so the endometrium may not always test positively for tuberculosis. Collecting specimens from multiple sites should be practiced to improve the yield. Chhabra et al stated that histopathology and bacteriology are both complementary to each other but are not reliable when singularly performed.[18]

\section{CONCLUSIONS}

There exists a lot of dilemma in diagnosis of genital tuberculosis even with the advent of newer diagnostic techniques. It is imperative to bear in mind the possibility of genital tuberculosis in women of reproductive age group, presenting with symptoms of infertility, chronic pelvic pain and menstrual irregularities, where other diagnoses have been excluded. If we only rely on PCR and AFB reports, as in this case, we may miss identifying a few cases. It should be borne in mind that where clinical picture resembles gestational tuberculosis, but the PCR and AFB do not indicate so, there is a need for further investigations, especially histopathology to confirm or exclude the diagnosis.

\section{REFERENCES}

[1] Aka N, Vural ZE. Evaluation of patients with active pulmonary tuberculosis for genital involvement. J Obset Gynaecol Res 1997;23(4):337-40.

[2] Güngördük K, Ulker V, Sahbaz A, et al. Postmenopausal tuberculosis endometritis. Infect Dis Obstet Gynecol 2007;2007:27028.

[3] Neonakis IK, Spandidos DA, Petinaki E. Female genital tuberculosis: a review. Scand J Infect Dis 2011;43(8):56472.

[4] Akbulut S, Arikanoglu Z, Basbug M. Tubercular tuboovarian cystic mass mimicking acute appendicitis: a case report. J Med Case Rep 2011;5:363.
[5] Health protection agency. Preliminary Annual Report on Tuberculosis Reported in 2002 England, Wales and Northern Ireland. London: HPA, 2004. [www.hpa.org.uk/infections].

[6] Rose AMC, Sinka K, Watson JM, et al. An estimate of the contribution of HIV infection to the recent rise in tuberculosis in England and Wales. Thorax 2002;57(5):442-5.

[7] Malhotra N, Malhotra J, Saxena R, et al. Jeffcoate's Principles of Gynaecology. Chapter - 22. Genital Tuberculosis. $9^{\text {th }}$ edn. Jaypee Brothers Medical Publishers 2018.

[8] Pfyffer GE, Wittwer F. Incubation time of mycobacterial cultures: How long is long enough to issue a final negative report to the clinician? J Clin Microbiol 2012;50(12):4188-9.

[9] Gogate S, Joshi S, Gogate A. Tubal factor in infertility endoscopic and microbiological evaluation. J Obstet Gynecol India 1994;44:282-5.

[10] Sweet RL, Mills J, Hadley KW, et al. Use of laparoscopy to determine the microbiologic etiology of acute salpingitis. Am J Obstet Gynecol 1979;134(1):68-74.

[11] Wolner-Hanssen P, Mardh PA, Svensson L, et al. Laparoscopy in women with chlamydial infection and pelvic pain: a comparison of patients with and without salpingitis. Obstet Gynecol 1983;61(3):299-300.

[12] Raut VS, Mahashur AA, Sheth SS. The Mantoux test in the diagnosis of genital tuberculosis in women. Int J Gynaecol Obstet 2001;72(2):165-9.

[13] Soltys MA. An anti-tuberculous substance in tuberculous organs. J Comp Pathol 1953;63(2):147-52.

[14] Bhanu NV, Singh UB, Chakraborty M, et al. Improved diagnostic value of PCR in the diagnosis if female genital tuberculosis leading to infertility. J Med Microbiol 2005;54(Pt 10):927-31.

[15] Thangappah RBP, Paramasivan CN, Narayan S. Evaluating PCR, culture \& histopathology in the diagnosis of female genital tuberculosis. Indian J Med Res 2011;134(1):40-6.

[16] Rozati R, Roopa S, Rajeshwari CN. Evaluation of women with infertility and genital tuberculosis. J Obstet Gynaecol India 2006;56:423-6.

[17] Restrepo BI, Gomez DI, Shipley GL, et al. Selective enrichment and detection of mycobacterial DNA in paucibacillary specimens. J Microbiol Methods 2006;67(2):220-9.

[18] Chhabra S, Narang P, Gupte N. A study of 150 cases of endometrial cultures for Mycobacterium tuberculosis. J Obstet Gynaecol India 1986;36(1):146-9. 